\title{
VARIABLES INFLUYENTES EN LA ELECCIÓN ENTRE CARRETERA Y FERROCARRIL PARA CARGA GENERAL DE COMERCIO EXTERIOR
}

\author{
Juan Diego Pineda-Jaramilloํ, Iván Sarmiento²
}

\author{
${ }^{1}$ Doctorado en Ingeniería Civil y Urbanismo. Docente de Ingeniería Civil \\ Correo electrónico: juapija1@doctor.upv.es \\ 2Ph.D. en Transporte. Profesor Departamento de Ingeniería Civil \\ Universidad Nacional de Colombia, sede Medellín
}

Recibido: 10 de mayo del 2014. Aprobado: 20 de julio del 2014.

Cómo citar este artículo: J. D. Pineda-Jaramillo y I. Sarmiento, "Variables influyentes en la elección entre carretera y ferrocarril para carga general de comercio exterior". Ingeniería Solidaria, vol. 10, n. ${ }^{\circ}$ 17, pp. 29-37, en.-dic., 2014. doi: http://dx.doi.org/10.16925/in.v9i17.792

Resumen. Aunque el transporte de carga por carretera presenta muchos inconvenientes en Colombia, no existe un estudio que demuestre cuáles son las variables que influyen en la elección de un modo para transportar carga, para saber si realmente sería útil un ferrocarril para una determinada región. En este estudio se realiza una investigación cualitativa a través de las técnicas de grupo focal con las empresas importantes de una región, ilustrándose la metodología con el caso de Antioquia; de esta manera, se logran conocer las variables que influyen en la elección de un modo de transporte por parte de las empresas que exportan e importan mercancías. Estas variables serán importantes para futuros trabajos de estimación de la demanda.

Palabras clave: transporte ferroviario, comercio exterior, preferencias declaradas, transporte de carga.

\section{Influential Variables in the Choice Between Roads and Railways for General Foreign Trade Cargo}

\begin{abstract}
Although cargo transport by road involves many difficulties in Colombia, there is no existing study showing the variables that influence the choice of a cargo transport modality, to determine whether or not a railway would truly be useful in a particular region. In this study, a qualitative investigation is performed using focus group techniques among important companies of a region, illustrating the methodology with the case of Antioquia; in this manner, the variables that influence the choice of transport modality by companies that export and import merchandise are determined. These variables will be important for future works when estimating demand.
\end{abstract}

Keywords: railway transport, foreign trade, declared preferences, cargo transport.

\section{VARIÁVEIS INFLUENTES NA ESCOLHA ENTRE RODOVIA F FERROVIA PARA CARGA GERAL DE COMÉRCIO EXTERIOR}

Resumo. Embora o transporte de carga por rodovia apresente muitos inconvenientes na Colômbia, não existe um estudo que demonstre quais são as variáveis que influenciam na escolha de um modo para transportá-la, para saber se realmente seria útil uma ferrovia para determinada região. Neste estudo, realiza-se uma pesquisa qualitativa por meio das técnicas de grupo focal com as empresas importantes de uma região e ilustrou-se a metodologia com o caso de Antioquia; dessa maneira, foi possível conhecer as variáveis que influenciam na escolha de um modo de transporte por parte das empresas que exportam e importam mercadorias. Essas variáveis serão importantes para futuros trabalhos de estimativa da demanda.

Palavras-chave: transporte ferroviário, comércio exterior, preferências declaradas, transporte de carga. 


\section{Introducción 1}

El transporte de carga fue quizá la razón por la cual se creó el ferrocarril, para mover materiales provenientes de la minería y, posteriormente, para la logística de la Guerra de Secesión estadounidense; este tipo de transporte fue siempre la razón que lo hizo evolucionar de la locomotora de vapor hasta las locomotoras de motor eléctrico con generador diésel. Desde la revolución industrial, el ferrocarril ha jugado un papel fundamental en el comercio y la industria al acortar distancias [1].

Los primeros ferrocarriles para transporte de carga se establecieron en los siglos XVII y XVIII, los cuales consistían en vagones manejados por caballos en las minas de carbón y canteras. La superficie de rodadura era de losa de piedra o de madera, obteniendo resultados insatisfactorios debido a que la carga se volvió más pesada [2].

En 1863, Colombia aprobó una nueva Constitución Política que dividió el territorio en nueve estados federales (Antioquia, Bolívar, Boyacá, Cauca, Cundinamarca, Magdalena, Panamá, Santander y Tolima), adoptando el nombre de Estados Unidos de Colombia. Por ese entonces, Panamá aún era una provincia colombiana. Precisamente, la construcción de ferrocarriles en Colombia comenzó con el ferrocarril de Panamá, entre esta provincia - sobre el Océano Pacífico-y los puertos de Colón — sobre el Océano Atlántico-, entre 1849 y 1854 [3]. No obstante, el ferrocarril en Panamá nada aportó a la economía y tecnología en Colombia -ni siquiera en el siglo XIX-. Es por eso que se puede decir que el primer ferrocarril que pudo llamarse colombiano fue el de Barranquilla a Puerto Colombia.

Después de la construcción del ferrocarril de Panamá, se construyeron muchos otros ferrocarriles - como el de Antioquia, Caldas, Cundinamarca, La Dorada, La Sabana y Tolima, Huila, entre otros- que fueron concebidos con un carácter local y con el fin de que se articularan a los ríos Cauca y Magdalena que estaban en condiciones de navegabilidad en ese momento. Esto permitió que Colombia contara con dinámicas internas adecuadas para el transporte multimodal de mercancías y pasajeros. Se puede añadir que los ferrocarriles en Colombia siempre fueron aumentando en longitud y capacidad conforme la producción y expor-

1 El presente artículo se deriva del trabajo desarrollado en la tesis de Maestría "Modelo de elección de modo de transporte entre carretera y ferrocarril para carga general susceptible a exportación-importación. Aplicación al corredor vial Medellín-Puerto de Cartagena", Universidad Nacional de Colombia, sede Medellín, 2012-2013 [18]. tación de café iba creciendo, pues de 1932 a 1943 aumentó su producción en un 33\% [4].

En la actualidad, el 70\% de la carga que se mueve en Colombia circula por carreteras, y aunque existen cerca de $160000 \mathrm{~km}$, sólo el 15\% de estas están pavimentadas. Por otro lado, el 30\% de la carga colombiana lo constituye el carbón movilizado en la costa Atlántica a través de $350 \mathrm{~km}$ de ferrocarril. Esto muestra que el ferrocarril con $1,5 \%$ de longitud respecto a la red pavimentada moviliza en Colombia más del $40 \%$ de la carga que se transporta en carretera, o sea, que un kilómetro de línea férrea en un entorno minero colombiano mueve más de 25 veces que un kilómetro promedio de red pavimentada, y aunque Colombia invirtiera 40 billones de pesos en el 2013 en autopistas, se sigue basando toda la movilización de la carga general en un solo modo sin seguir la opción más eficiente. Por lo anterior, es innegable el impacto negativo que tiene en la economía el atraso vial del país, y en general, las muchas falencias que tienen en Colombia todos los sistemas de transporte de carga.

Colombia no cuenta con un sistema ferroviario eficiente, sino tan sólo con unos fragmentos de lo que este debería ser. Los más utilizados son los $193 \mathrm{~km}$ que unen la mina Pribennow - en La Loma, departamento del Cesar- y el Puerto de Drummond Ltd. - en Santa Marta, departamento del Magdalena-, que hacen posible el transporte y la exportación de carbón por el tramo conocido como La Loma - Santa Marta. También existen $150 \mathrm{~km}$ que unen la mina de carbón del Cerrejón, con su respectivo puerto de embarque. Cabe señalar que en esos casi $350 \mathrm{~km}$ se transporta alrededor del 30\% de la carga movilizada en Colombia. Esto demuestra la importancia del ferrocarril en el transporte de carga, aunque en este caso sólo se esté utilizando para un mineral.

Aunque el ferrocarril se destaca sobre otros modos de transporte de carga terrestre por su rendimiento en el transporte de mercancías a larga distancia (comparado con el camión, el ferrocarril necesita de un corredor de menor ancho y altera menos el entorno), es conveniente destacar que la selección de un modo de transporte desde el punto de vista de los tomadores de decisiones es un problema complejo, que abarca distintos actores y engloba distintas variables.

Así pues, este artículo pretende analizar el problema de la identificación de las variables más influyentes en la elección de un modo de transporte de carga, sean variables cuantitativas o cualitativas, por parte de los tomadores de decisiones, para el caso de la construcción de un corredor ferroviario entre una ciudad 
capital y un puerto a más de $500 \mathrm{~km}$ de distancia de la misma, y donde actualmente sólo existe una carretera que los comunica. Esta investigación se limita a la carga general de comercio exterior antioqueña, y la metodología propuesta se aplicará al caso de un corredor en Colombia, entre Medellín y el Puerto de Cartagena.

En la segunda parte de este artículo, se presenta el estado del arte. En la tercera, se presenta la metodología empleada. En la cuarta sección, se presenta el caso de estudio. Los resultados se muestran en la quinta parte. En la sexta sección, se presenta la discusión de estos resultados, y finalmente, en el séptimo apartado, se muestran las conclusiones y recomendaciones para trabajos futuros.

\section{Estado del arte}

Uno de los retos que tiene el transporte de carga en países latinoamericanos es la determinación de las variables que influyen en la elección por parte de los tomadores de decisiones de las empresas, al decidir entre enviar una carga por carretera o por ferrocarril -en caso de que exista-, más aún cuando los sistemas ferroviarios no funcionan adecuadamente en muchos casos.

Los proyectos de rehabilitación de redes ferroviarias se hacen difíciles de justificar sin un estudio pertinente de este problema de la elección, de modo que permita estimar la demanda futura de proyectos ferroviarios.

Este problema también se relaciona con la sostenibilidad de las carreteras, las cuales, ante un exagerado flujo de carga, pueden sufrir consecuencias globales negativas en cuanto al mantenimiento de las mismas - fatiga de los pavimentos-, así como con los usuarios, por el aumento en el consumo de combustible, el tiempo de viaje - costos de operación- y costos de mantenimiento de vehículos; también influye en la sociedad, por el incremento de las externalidades de tipo ambiental y de seguridad vial. Por todo ello, varios autores demuestran lo conveniente del uso del ferrocarril de carga como sustituto y también como complemento a la carretera [5]-[8].

El transporte de carga por carretera presenta muchos problemas en Colombia, como altos costos de operación, gran impacto negativo en el medio ambiente, fatiga acelerada de los pavimentos a causa de los camiones de carga, altos índices de accidentalidad en las vías, elevado consumo de combustible, altos tiempos de viaje, etcétera. En la academia y la industria se habla de la ingente necesidad de un sistema ferroviario de carga en Colombia para dinamizar la economía nacional, pero no hay evidencia en material consultado de un estudio pertinente del tema. Además, desde el 2012, con la entrada en vigencia del Tratado de Libre Comercio entre Colombia y Estados Unidos cobra mayor importancia un estudio de esta naturaleza, ya que Colombia carece de una adecuada infraestructura para competir.

En países como Estados Unidos, Australia, Rusia y otros países europeos, se han utilizado diferentes variables por parte de los tomadores de decisiones para elección modal, usando variables de todo tipo, socioeconómicas y latentes.

Tal es el caso de Dinamarca, donde para la distribución modal de carga entre cinco modos se tienen en cuenta diversas variables relacionadas con el costo y el tiempo, incluyendo en este último, el tiempo de viaje, tiempo de congestión en carretera, tiempo de espera, entre otros [9].

Otro es el caso en que las variables tenidas en cuenta, de una manera empírica para la elección modal, son la frecuencia, distancia, restricciones de circulación, costo, tiempo de viaje, daño que puede sufrir la carga, retrasos, entre otros. Aplicado a la carga exterior española por el puerto de Valencia [10].

Siguiendo con ejemplos del mismo tipo, se tiene el caso francés, en el que se encuentra que la distancia, la accesibilidad al modo de transporte, las instalaciones del transportista y la forma de envío, son los determinantes críticos en la elección de modo de transporte. De nuevo, la elección de las variables tenidas en cuenta para la elección modal se realiza de manera empírica [11].

A pesar de estos análisis, en Colombia, no hay evidencias de un estudio pertinente para saber cuáles son las variables influyentes desde el punto de vista de los empresarios que toman la decisión, en la elección de un modo de transporte de carga para corredores, donde la carretera podría competir con un posible ferrocarril.

\section{Metodología}

La metodología seguida para la determinación de variables influyentes en la elección entre carretera y un hipotético sistema férreo fue la siguiente:

1. Identificar los productos de comercio exterior que son transportados entre la ciudad y el puerto en estudio, dividiendo inicialmente los productos por tipología y determinando el porcentaje de cada tipo de carga con relación al comercio exterior, y así determinar el tipo de carga predominante en el corredor. 
2. Identificar las empresas que exportan e importan dichos productos, reconociendo aquellas que transportan una mayor cantidad de carga. Cabe destacar que generalmente un pequeño porcentaje de empresas que no supera el 20\% transporta más del $80 \%$ de la carga, este principio se conoce como "principio de Pareto" [12].

3. Contactar un grupo de responsables de logística o tomadores de decisión de esas empresas identificadas para realizar un grupo focal.

4. Conformar un grupo focal, con el que se puedan identificar las variables influyentes en la elección modal. Un grupo focal puede definirse como una discusión cuidadosamente diseñada para obtener las percepciones sobre una particular área de interés [13]. La evidencia de los grupos focales sugiere que las opiniones de un individuo pueden cambiar en el curso de la discusión con otros, porque las personas influyen las unas a las otras por medio de sus comentarios. Los analistas de grupos focales pueden descubrir más información sobre cómo dicho cambio ocurre y la naturaleza de los factores influyentes. De esta manera, el científico tiene la posibilidad de influir en el cambio por voluntad propia [13]. Estos grupos deben ser lo suficientemente pequeños como para permitir la oportunidad a cada participante de compartir su discernimiento de las cosas, y a la vez lo suficientemente grande como para proveer diversidad de percepciones, un número adecuado es entre 6 a 12 participantes [14].

\section{Caso de estudio}

Para iniciar, es importante definir la carga general como aquella que está envuelta o sin envoltura, se puede tratar como una unidad. Se transporta en cajas, cuya forma, peso y dimensiones se ajustan a las características propias de esta. Algunos productos de este tipo son: zapatos, ropa, tela, computadoras, manufacturas en general, entre otras. [15]. Igualmente, la carga general se divide en:

- Carga general fraccionada: son los bienes sueltos o individuales, por ejemplo: paquetes, sacos y cajas.

- Carga general unitarizada: son artículos individuales agrupados en unidades, como contenedores.

\subsection{Carga en Colombia}

La movilización de carga de un país indica su nivel de desarrollo, su nivel de productividad y su índice de competitividad. En 2009, Colombia tenía cerca de 45 millones de habitantes (75\% viviendo en áreas urbanas) y más de un millón de kilómetros cuadrados de superficie.

Tabla 1. Movilización de carga en Colombia (en toneladas)

\begin{tabular}{|c|c|c|c|c|c|c|c|c|}
\hline \multirow{2}{*}{ Año } & \multirow{2}{*}{ Carretero } & \multicolumn{3}{|c|}{ Férreo } & \multirow{2}{*}{ Fluvial } & \multirow{2}{*}{ Aéreo } & \multirow{2}{*}{ Cabotaje } & \multirow{2}{*}{ Total } \\
\cline { 3 - 6 } & & Concesiones & Carbón & Total férreo & & & & \\
\hline 1994 & 82841000 & 812000 & 12833000 & 13645000 & 2890000 & 140000 & 3700000 & 103216000 \\
\hline 1995 & 86741000 & 882000 & 13734000 & 14616000 & 2634000 & 140000 & 4000000 & 108131000 \\
\hline 1996 & 71168000 & 981000 & 15354000 & 16335000 & 3062000 & 142000 & 4324000 & 95031000 \\
\hline 1997 & 89399000 & 836000 & 16370000 & 17206000 & 2755000 & 139000 & 3997000 & 113496000 \\
\hline 1998 & 84350000 & 779000 & 21842000 & 22621000 & 3049000 & 119000 & 4009000 & 114148000 \\
\hline 1999 & 77674000 & 367000 & 25035000 & 25402000 & 3735000 & 134000 & 1385000 & 108330000 \\
\hline 2000 & 73034000 & - & 31170000 & 31170000 & 3802000 & 100000 & 797000 & 108903000 \\
\hline 2001 & 100284000 & - & 33457000 & 33457000 & 3069000 & 104000 & 720000 & 137634000 \\
\hline 2002 & 84019000 & - & 31032000 & 31032000 & 3480000 & 122000 & 532000 & 119185000 \\
\hline 2003 & 99782000 & 37000 & 42744000 & 42781000 & 3725000 & 132000 & 928000 & 147348000 \\
\hline 2004 & 117597000 & 317000 & 45865000 & 46181000 & 4211000 & 129000 & 588000 & 168706000 \\
\hline 2005 & 139646000 & 308000 & 48919000 & 49227000 & 4863000 & 135000 & 400000 & 194271000 \\
\hline 2006 & 155196000 & 314000 & 49394000 & 49708000 & 4025000 & 138000 & 509000 & 209576000 \\
\hline 2007 & 183126000 & 375000 & 52829000 & 53204000 & 4562000 & 137000 & 454000 & 241484000 \\
\hline 2008 & 169714000 & 236000 & 58236000 & 58472000 & 4953000 & 123000 & 372000 & 233634000 \\
\hline 2009 & 173558000 & 254000 & 59144000 & 59398000 & 4070000 & 109000 & 364000 & 237499000 \\
\hline
\end{tabular}

Fuente: [16] 
En la tabla 1 se observa la carga movilizada en Colombia, en el período 1994-2009. En esos 15 años se ha dado un crecimiento del $210 \%$ en el tonelaje movilizado por carretera; un $435 \%$ en el trasportado por ferrocarril debido al incremento del transporte de carbón; un 171\% en el fluvial hasta el 2008 cuando empezó a decaer; por su parte, el aéreo se ha mantenido más o menos constante, y por último, el de cabotaje ha disminuido a la décima parte de lo que movilizaba quince años antes del 2009. A 2009, Colombia movilizaba 237 millones de toneladas (sin incluir ductos) y su distribución por modo era de $73,10 \%$ en carretera, $25,00 \%$ en ferroviario, $1,70 \%$ en fluvial, $0,05 \%$ en aéreo y $0,15 \%$ en cabotaje.

En cuanto a la carga de comercio exterior, durante el primer semestre del año 2009, se movilizaron por los cuatro principales puertos marítimos colombianos (Barranquilla, Buenaventura, Cartagena y Santa Marta), 30,9 millones de toneladas (cerca del 18\% de la carga por carretera) incluyendo graneles y carga refrigerada, sin incluir carbón que se mueve mayoritariamente por ferrocarril y a través de puertos especializados [17]. El desagregado se presenta en la tabla 2.

Tabla 2. Importaciones y exportaciones de Colombia, primer semestre de 2009

\begin{tabular}{|l|l|l|}
\hline \multicolumn{1}{|c|}{ Tipo de Carga } & \multicolumn{1}{c|}{ Toneladas } & Participación \\
\hline Carga general & 5529540 & $17,89 \%$ \\
\hline Graneles líquidos & 3757539 & $12,16 \%$ \\
\hline Graneles sólidos & 21166292 & $68,50 \%$ \\
\hline Refrigerados & 448404 & $1,45 \%$ \\
\hline Gran total & 30901776 & $100 \%$ \\
\hline
\end{tabular}

Fuente: [17]
La carga general por puerto de entrada/salida es de 5.5 millones de toneladas y la distribución por puerto es la mostrada en la tabla 3 :

Tabla 3. Carga general de Colombia, primer semestre 2009 por puerto

\begin{tabular}{|l|l|l|l|}
\hline \multicolumn{1}{|c|}{ Puerto } & \multicolumn{1}{c|}{ Total TEU } & $\begin{array}{c}\text { Carga general } \\
\text { (toneladas) }\end{array}$ & Participación \\
\hline Barranquilla & 36115 & 941004 & $17 \%$ \\
\hline Buenaventura & 191899 & 2229392 & $40 \%$ \\
\hline Cartagena & 223123 & 2202391 & $40 \%$ \\
\hline Santa Marta & 3849 & 156753 & $3 \%$ \\
\hline Total & 454986 & 5529540 & $100 \%$ \\
\hline
\end{tabular}

Fuente: [17]

\subsection{Carga en Antioquia}

Antioquia es el segundo departamento más poblado de Colombia con cerca de 6 millones de habitantes en 2009 (15\% de Colombia) y una superficie de $63 \mathrm{mil}$ kilómetros cuadrados (5,5\% de Colombia). La tabla 4 muestra la carga de comercio exterior de Antioquia en el primer semestre del 2009.

El tonelaje total de comercio exterior de Antioquia representa el 6\% del tonelaje total de comercio exterior movilizado en Colombia. Si se tiene en cuenta únicamente la carga general, entonces la participación de Antioquia respecto al país asciende al $12 \%$.

Por otra parte, si se tiene en cuenta que el PIM de Antioquia es de casi $20 \%$ respecto al de Colombia, se concluye que los productos antioqueños son de mayor valor unitario que el promedio del país, considerando

Tabla 4. Carga de comercio exterior de Antioquia, primer semestre de 2009

\begin{tabular}{|l|c|c|c|c|c|c|c|c|c|}
\hline \multirow{2}{*}{$\begin{array}{c}\text { Tipo de } \\
\text { Carga }\end{array}$} & \multicolumn{2}{|c|}{ Importaciones } & \multicolumn{2}{c|}{ Exportaciones } & \multicolumn{3}{c|}{ Total } & \multicolumn{3}{c|}{ Distribución por puerto de entrada/ } \\
salida
\end{tabular}

Fuente: [17] 
que los graneles líquidos y sólidos tienen menor valor unitario que la carga general, además de que productos como el carbón y el petróleo no se producen en tanta cantidad en el departamento como en otras regiones (la carga de graneles sólidos de comercio exterior en Antioquia es apenas el 5\% del respectivo ítem a nivel nacional).

Los principales productos de importación de Antioquia, en cuanto a carga general se refiere, son: hierro, acero, lámina, dimetil tereftalato, pulpa de madera, químicos orgánicos e inorgánicos, plástico, automóviles y autopartes; los principales productos de exportación de Antioquia son: vidrio, café, hojalata, láminas, abonos, papel, plástico, galletas, químicos orgánicos e inorgánicos.

La distribución de la carga general se concentra más en Cartagena, que ha sido el puerto tradicional para Antioquia [17].

Haciendo un análisis de la tabla 4, se tiene:

- En importaciones, el comercio antioqueño movilizó 470562 toneladas en carga general.

- Mientras que en exportaciones el comercio antioqueño movilizó 194945 toneladas en carga general.

- De las 665507 toneladas de carga general del comercio antioqueño que se movilizó por el puerto de Cartagena, el 70,7\% (470 562 toneladas) corresponde a carga de importación, mientras que el 29,3\% (1949 945 toneladas) corresponde a carga de exportación [17].

Proyectando al año 2013, el comercio antioqueño movilizó aproximadamente 1500000 toneladas correspondientes a carga general anual.

Tomando los mismos porcentajes para exportación-importación, se tiene que el 70,7\% (1 060500 toneladas) será carga de importación mientras que el 29,3\% (439 500 toneladas) será carga de exportación.

El escenario presentado es conservador, puesto que sólo se está considerando la carga de comercio exterior como una parte del universo potencial en el que se piensa captar una parte para el ferrocarril, y no se está considerando la carga interna entre regiones del país, como por ejemplo la de Antioquia a Córdoba, a
Sucre y a Bolívar, así como de esos departamentos hacia Antioquia y entre ellos.

\subsection{Grupo focal}

Como se dijo anteriormente, el grupo focal contó con la colaboración de las empresas generadoras/receptoras de carga de la región. La ejecución del grupo focal se realizó en la Universidad Nacional de Colombia, el 20 de abril de 2012.

Los objetivos de este grupo focal eran:

- Identificar las variables influyentes en la elección de un modo de transporte de carga en Antioquia.

- Conocer la opinión de los asistentes sobre un posible sistema ferroviario en la región antioqueña.

El grupo focal fue conformado por 8 personas encargadas del área de transporte y comercio exterior de cuatro de las empresas que más mueven carga de comercio exterior en la región con un 5,6\% de la carga importada y un 23,3\% de carga exportada total del departamento de Antioquia. El número de asistentes es adecuado para un grupo focal. Además, este fue moderado por una comunicadora para garantizar la imparcialidad en su ejecución y evitar el sesgo por falta de independencia de algún miembro o del moderador.

La tabla 5 muestra la caracterización de los participantes del grupo focal.

Tabla 5. Caracterización de los participantes del grupo focal

\begin{tabular}{|c|c|c|}
\hline Participantes & Empresa & Cargo \\
\hline 1 & OMYA & Jefe de logística y compras \\
\hline 2 & \multirow[b]{2}{*}{ Cristalería Peldar S.A. } & Área de compras \\
\hline 3 & & $\begin{array}{l}\text { Coordinador de logística } \\
\text { de planta de Envigado }\end{array}$ \\
\hline 4 & \multirow[t]{2}{*}{ Sofasa } & $\begin{array}{l}\text { Responsable de transporte } \\
\text { en Sofasa }\end{array}$ \\
\hline 5 & & Distribución de vehículos \\
\hline 6 & \multirow{3}{*}{ Productos Familia } & $\begin{array}{l}\text { Comercio exterior de la } \\
\text { compañía }\end{array}$ \\
\hline 7 & & $\begin{array}{l}\text { Transporte nacional } \\
\text { terrestre }\end{array}$ \\
\hline 8 & & $\begin{array}{l}\text { Jefe de transporte } \\
\text { internacional }\end{array}$ \\
\hline
\end{tabular}

Fuente: elaboración propia 


\section{Resultados}

A continuación, se presenta un resumen general de los resultados obtenidos en el grupo focal. Aunque había ocho personas, hacían parte de cuatro empresas, por lo que este resumen tiene en cuenta lo que respondía en general cada empresa.

Pregunta 1: ¿en qué modos de transporte movilizan la carga de exportación-importación desde la empresa hacia el puerto y viceversa, y qué variables o criterios tienen en cuenta para elegir dicho modo?

En general, todos transportan la carga de la empresa a los puertos, y viceversa, en contenedores movilizados por tracto camiones.

La variable que tienen en cuenta a la hora de elegir dicho sistema de transporte es básicamente el costo.

Se hace mucho énfasis en que no les gusta hacer muchos transbordos con la carga porque esto genera más costos. Mientras más cargues y descargues tenga un contenedor, más costoso se convierte el flete.

También se menciona que se utiliza en menor porcentaje el sistema multimodal a través del río.

Sobre el tiempo de viaje, se descubrió que no es una variable muy influyente en su elección de modo de transporte ya que el tiempo que se ahorrarían eligiendo otro modo de transporte lo perderían en el puerto debido a su ineficiencia logística. Este factor tiempo sólo es importante cuando la carga ya está en el puerto.

Pregunta 2: ¿qué factores cree usted que son los que influyen en el transporte de carga en Antioquia?

En la región es muy importante la variable costo, ya que como la región es una gran importadora de carga, pero la exportación cae sobre unos pocos generadores, entonces según la temporada del año los generadores de carga tratan de enviar más carga a los puertos, ya que ven una oportunidad de ahorro de costos porque hay muchos camiones yendo vacíos hacia los puertos a buscar carga importada.

Aparte del costo que todas las empresas están midiendo en todo tiempo, se mira también la variable "frecuencia" y "disponibilidad" de los medios de transporte.

Otra variable importante es la cantidad de nodos que tiene la cadena modal de transporte, mientras más se pueda minimizar el número de transbordos que haga la carga, mejor va a ser el transporte de carga en términos de costo y de tiempo, porque cada transbordo es un costo adicional.

Otra variable determinante a la hora de elegir un modo de transporte es la infraestructura que ofrece el modo de transporte; si no se cuenta con la infraes- tructura necesaria para llenar un contenedor en sus terminales, no será competitivo el modo de transporte.

Muchas de estas falencias se deben a la falta de parques logísticos en el Valle de Aburrá, y la ubicación de la industria, ya que por esto los camiones deben recorrer mucha distancia para conseguir los contenedores y poder cargarlos. Se debe estudiar dónde ubicar un parque logístico de contenedores para combatir esta problemática.

Pregunta 3: ¿considera que en Antioquia hace falta un ferrocarril? ¿Por qué?

Todos están de acuerdo en que de existir otro medio de transporte para carga, debería ser un medio de transporte complementario a los ya existentes, y que no compitan entre sí.

Un sistema ferroviario sería muy útil en la región, ya que su capacidad de carga es enorme en comparación con la capacidad de carga por vía terrestre.

Para pensar en un sistema ferroviario, la academia debe ponerse a pensar en una proyección de lo que va a ser Antioquia en los próximos años.

Además del ferrocarril, sería importante que los puertos mejoren su eficiencia, para no tener tiempos muertos allí.

Pregunta 4: si existiera un ferrocarril entre Medellín y el puerto al que usted recibe/envía carga, ¿bajo qué criterios lo utilizaría?

El ferrocarril lo utilizarían siempre y cuando fuera más económico que el transporte terrestre.

Otra variable que se tendría en cuenta sería la frecuencia del servicio.

Las industrias buscan minimizar costos a toda costa, y si el ferrocarril los redujera, seguramente sí sería utilizado.

Para que este sistema sea eficiente, debe haber una demanda de carga bastante alta, porque si una empresa sólo mueve tres contenedores al mes, no les es rentable moverlas por este sistema. Todo radica en las frecuencias del servicio y en su costo.

Para que un sistema ferroviario progrese en la región, se debe educar a los empresarios.

Pregunta 5: ¿cuál cree usted que son los motivos por los que no progresan los proyectos de construcción de ferrocarriles para transporte de carga en el país?

Existen plataformas logísticas mal ubicadas, todas las acciones que se toman son con intereses políticos, todo está totalmente direccionado y no se tienen en cuenta todos los actores del transporte.

Existen estudios de dónde ubicar puertos secos, dónde desarrollar transporte multimodal, falta actuar. 
Existen muchos intereses individuales que perjudican las propuestas de ferrocarriles, ya que no les interesa que exista uno. Actores influyentes en el transporte de carga en el país, como los transportadores y los puertos, mientras sigan teniendo las mismas condiciones y la misma rentabilidad que en este momento tienen, no van a apoyar proyectos de ferrocarriles en el país.

Pregunta 6: ¿qué falencias ve usted en el transporte de carga por carretera en Antioquia? y ¿qué recomendaría para mejorar la situación?

En esta pregunta, los participantes respondieron resumiendo todo lo que hablaron durante todo el grupo focal. Mencionaron, a modo de resumen, que existen problemas de integración multimodal, faltan vías, parques logísticos.

Falta que el Gobierno implemente una cultura logística en el país, tener un pensamiento de nación y región, más que de utilidades individuales.

De las respuestas obtenidas por los participantes del grupo focal, se puede identificar que las variables influyentes en la elección entre carretera y ferrocarril para carga general de comercio exterior en Antioquia son costo y frecuencia de servicio. Siempre y cuando el sistema férreo sea más económico y ofrezca una frecuencia de servicio competitiva respecto al transporte carretero, tal como está estipulado en la presente propuesta, todas las empresas exportadoras-importadoras de carga por el puerto de Cartagena serían potenciales clientes.

\section{Discusión}

En el presente estudio solo fue considerada la carga general, debido a que se pretendía enfocar el trabajo en un solo tipo de carga para analizar profundamente a los empresarios y su posición frente al transporte de carga en Colombia. Aun así, es atrevido afirmar que el pensamiento de estos se puede extrapolar al resto de empresas que manejan todas las tipologías de carga en Colombia.

Los costos promedio para transportar una carga de exportación son menores que los costos para importación; esto es porque Antioquia importa mucha más carga de la que exporta, entonces muchas veces los camiones de carga, para no tener que volver vacíos a los puertos, cobran más barato los fletes para regresar al puerto; además, los costos de transporte son muy elevados en el país por muchas razones; por ejemplo, en promedio, en Estados Unidos o Europa, un camión re- corre 20 000-22 000 kilómetros al mes; en cambio, en Colombia, está recorriendo máximo 12000 kilómetros al mes. Las vías en Antioquia no tienen una calidad óptima, esto encarece el flete terrestre.

Uno de los retos existentes en Colombia es el mejoramiento de las cadenas logísticas; esto puede lograrse mediante el diseño de corredores logísticos para el transporte de carga.

\section{Conclusiones y recomendaciones}

Las variables influyentes en la elección entre carretera y ferrocarril para transportar carga general de comercio exterior en Antioquia son costo y frecuencia de servicio. Siempre y cuando existan campañas de educación para los empresarios de la región, que muestren otras opciones efectivas como lo sería el ferrocarril, el empresario antioqueño arraigado al transporte carretero empezará a considerarlas. El ferrocarril propuesto entre Medellín y Cartagena, sobre el cual se preguntó en el grupo focal, es un buen ejemplo de ello.

Mucho se especula en el medio que al existir un ferrocarril para transporte de carga podría dejar muchos conductores de camiones sin trabajo, pero si existiese un ferrocarril, aumentaría la oferta de servicio, se minimizarían los costos y las empresas tendrían otra opción de transporte para movilizar su carga. Al minimizar los costos, bajaría el precio de los productos, por lo que podría existir una mayor demanda de producción. Esto, sumado a los actuales TLC que ha firmado el país con EE.UU., países europeos y países asiáticos, aumentaría la carga que habría que movilizarse en el país y, por tanto, se aumentaría la frecuencia del servicio de los ferrocarriles; a su vez, representaría mayor mano de obra necesaria y mayor cantidad de puestos de trabajo, por lo que los camioneros tendrían nuevas opciones de trabajo para llevar carga al ferrocarril o incluso de reconversión laboral.

Un buen sistema de transporte ferroviario en el país emplearía ferrocarriles para movilizar mayor cantidad de carga y dispondría de los camiones para distribuciones en distancias más cortas (no aplican los productos frágiles que deban seguir transportándose por camión, sino los que permitan un transporte multimodal). Esto traería consigo grandes beneficios para reducir la fatiga en los pavimentos y aumentar su vida útil, se minimizaría el deterioro acelerado de las vías y así aumentaría el presupuesto para pavimentar más carreteras en el país o se aumentaría la vida útil de las existentes. Aparte, al 
poder movilizar más fácil y rápidamente los productos del interior del país a los puertos, los costos de transporte de los productos se verían reducidos, y el mercado colombiano sería más competente.

Para terminar, hay que enfatizar en que un país sin inversión en un sistema moderno de transporte en todos sus modos, está condenado a ser económicamente incompetente.

Como trabajos complementarios, se propone realizar una modelación de la elección de modo para transporte de carga general entre carretera y ferrocarril considerando las variables aquí encontradas (costo y frecuencia), así como explorar la posibilidad de utilizar las vías ferroviarias en Colombia, no sólo para el transporte de carga, sino también para el transporte de pasajeros, sobre todo en corredores donde la carga las viabilice y la topografía permita conectar ciudades con un mercado potencial de viajes entre ellas.

También se propone, como trabajo posterior, ahondar sobre los mecanismos de regulación del modo de transporte férreo, que puede ser uno de los grandes obstáculos en la selección de este modo.

\section{Agradecimientos}

Los autores agradecen a Colciencias por financiar la presente investigación enmarcada en el Programa Jóvenes Investigadores e Innovadores 2011 "Virginia Gutiérrez de Pineda”.

\section{Referencias}

[1] C. F. Bonnett, Practical Railway Engineering, 1st ed. London: Imperial Collage Press, 1996.

[2] C. F. Bonnett, Practical railway engineering, 2nd ed., London: Imperial College Press, 2005.

[3] G. Poveda Ramos, "El primer ferrocarril en Colombia", DYNA, vol. 69, n. ${ }^{\circ} 137$, pp. 61-73, 2002. [En línea] Disponible en: http://www.redalyc.org/pdf/496/49613707. pdf

[4] G. Poveda Ramos, Ingeniería e Historia de las Ciencias, vol. V, Historia Social de la Ciencia en Colombia. Bogotá: Tercer Mundo Editores, 1993.

[5] Federal Railroad Administration, "Freight rail overview. U.S. Department of Transportation”. [En línea]. Disponible en: https://www.fra.dot.gov/Page/P0528. [Último acceso: en. 08 de 2014]
[6] D. S. Lawyer, "Rail vs. Truck Energy Efficiency". Oct. 2006. [En línea]. Disponible en: http://www.lafn. org/ dave/trans/energy/rail_vs_truckEE.html\#s4. [Último acceso: jun. 04 2014].

[7] D. J. Forkenbrock, "Comparison of external costs of rail and truck freight transportation", Transportation Research Part A: Policy and Practice, vol. 35, pp. 321-337, 2001.

[8] E. Pons, "Influencia futura del ferrocarril en el comercio exterior", Tesina de Especialización, Dep. d'Infraestruc. del Trans. i del Territ., Univ. Politècnica de Catalunya. Barcelona, 2011. [En línea] Disponible en: http:// upcommons.upc.edu/pfc/bitstream/2099.1/12538/1/ Influencia $\% 20$ ferrocarril $\% 20$ sobre $\% 20$ comercio $\% 20$ exterior_bo.pdf

[9] J. Rich, P. M. Holmblad y C. O. Hansen, "A weighted logit freight mode-choice model", Transportation Research Part E: Logistics and transportation review, $\mathrm{n}^{\circ} 45$, pp. 1006-1019, 2009.

[10] L. García, I. Martínez y D. Piñero, "Determinants of mode choice between road and shipping for freight transport. Evidence of four spanish exporting sectors", Journal of transport economics and policy, no 38, pp. 447-466, 2004.

[11] F. Jiang, P. Johnson y C. Calzada, "Freight demand characteristics and mode choice: An analysis of the results of modeling with disaggregate revealed preference data", Journal of transportation and statistics, vol. 2, n. ${ }^{\circ}$ 2, pp. 149-158, 1999.

[12] V. Pareto, Cours d'Economie Politique, Geneva: Droz, 1896.

[13] R. A. Krueger, Focus groups: A practical guide for applied research, Sage, California: Beverly Hills, 1991.

[14] T. Reyes, "Métodos cualitativos de investigación: los grupos focales y el estudio de caso", Forum Empresarial, vol. 4, n. ${ }^{\circ}$ 2, pp. 75-87, 2000.

[15] Cargo Week Americas. Expo carga. [En línea]. Disponible en: http://cargainfo.com/front_content.php?idcat $=1231$ \&idart=2740. [Último acceso: abr. 14 2014].

[16] Oficina Asesora de Planeación. Grupo Planificación Sectorial. Ministerio de Transporte de Colombia, Anuario Transporte en cifras. Estadísticas 2012, Bogotá, 2012. [En línea]. Disponible en: file://C:/Users/ admin/Downloads/Transporte\%20Cifras\%20-\%20Estadisticas\%202012.pdf

[17] L. Bustamante, Informe sobre comercio exterior Antioquia primer semestre 2009, Medellín, 2009.

[18] J. D. Pineda-Jaramillo, "Modelo de elección de modo de transporte entre carretera y ferrocarril para carga general susceptible a exportación - importación. Aplicación al corredor vial Medellín-Puerto de Cartagena”, Tesis de maestría. Dept. Ing. Civ., Univ. Nac. de Colombia, Medellín, 2013. 